\title{
Journal of Counseling Psychology Principal Reviewers, Contributing Reviewers and Pre-Doctoral Co-Reviewers (September 2016 to September 2017)
}

\section{PRINCIPAL REVIEWERS}

The Editor and Associate Editors wish to thank the following persons who served as principal reviewers for Journal of Counseling Psychology by reviewing a minimum of four manuscripts.

LaRae M. Jome

Maureen E. Kenny

Stefanía Ægisdóttir Mary Z. Anderson Alexis V. Arczynski

David Atkins

Scott A. Baldwin

Kira Banks

Jameta Barlow

Lynette H. Bikos

Sha'Kema Blackmon

Walter Buboltz

Stephanie L. Budge

Gary Burlingame

Grace I. L. Caskie

Edward C. Chang

MIke Cheung

Na-Yeun Choi

Brian Cole

Stephen W. Cook

Susan X. Day

Edward A. Delgado-Romero

David M. Dunkley

Robert Elliott

Nicole Else-Quest

Melissa Baker
Selda Celen-Demirtas
Hadar Ertl

Hadar Ertl

$\mathrm{Xu} \mathrm{Li}$

Ryon C. McDermott

Uma Chandrika Millner

Mike Schaub

\section{CONTRIBUTING REVIEWERS}

Thane Erikson

Fernando Estrada

Ann R. Fischer

Mary Fitzpatrick

Nadya A. Fouad

Myrna Friedlander

M. Paz Galupo

Garren Gaut

Omar Carlo Gioacchino Gelo

Marie S. Hammond

Laurie Heatherington

Mark J. Hilsenroth

Michiyo Hirai

Zhi-Jin Hou

Carla D. Hunter

Arpana G. Inman

Margo A. Jackson

Ae Kyung Jung

Sabra L. Katz-Wise

Anita E. Kelly

Gulsah Kemer

Franziska Kühne
Daniel G. Lannin

Ronald F. Levant

Chris Liang

William Ming Liu

Kenneth Locke

Yun Lu

P. Priscilla Lui

Brent Mallinckrodt

Suzanne E. Mazzeo

Ethan H. Mereish

Michael Mobley

Debbie Moskowitz

Nancy L. Murdock

Kevin L. Nadal

Helen A. Neville

Gary Peterson

David Pincus

Jae Puckett

Ashley K. Randall

Lance M. Rappaport

Robert J. Reese
Pål Gunnar Ulvenes

James Rounds

Pamela Sadler

Eleanor K. Seaton

William Sedlacek

Jena Shaw

Brad Shuck

Andy Siddaway

Stephanie Simon-Dack

Christopher Slaten

Lisa Beth Spanierman

Joel Steele

Lara M. Stepleman

Martin Swanbrow Becker

Eric Swank

Dawn M. Szymanski

Christina Thai

Alisia G. T. T. Tran

Kenneth T. Wang

Chiachih D. C. Wang

Russell Thomas Warne

Laurel B. Watson

Florian Weck

Serena Wee

Meifen Wei

Katharina Weitkamp

\section{CONTRIBUTING PRE-DOCTORAL CO-REVIEWERS}
Kritzianel Fisher
Majel Heath
Elliot Heath
Jackson Lipsitz

Marlene Merced
Liza Meredith
Patrick Painter
Ilana Seidman

Marlene Merced

Ilana Seidma

\author{
Leslie Spengler \\ Andrew Stapley \\ Patrick Williams \\ Christine Wolfer
}

